\title{
PADRÕES ANTROPOMÉTRICOS DE PESSOAS HIPERTENSAS
}

\section{ANTHROPOMETRIC PATTERNS OF HYPERTENSIVE INDIVIDUALS}

\section{PADRONES ANTROPOMÉTRICOS DE PERSONAS HIPERTENSAS}

\author{
Cláudia Geovana da Silva Pires ${ }^{1}$ \\ Maiara da Silva Brandão Rodrigues ${ }^{2}$ \\ Alana de Souza Reis Carneiro ${ }^{2}$ \\ Igor Fenando Lopes Assis ${ }^{3}$
}

Como citar este artigo: Pires CGS, Rodrigues MSB, Carneiro ASR, Assis IFL. Padrões antropométricos de pessoas hipertensas. Rev baiana enferm. 2018;32:e27997.

\begin{abstract}
Objetivo: descrever os padrões antropométricos de adultos hipertensos e caracterizar os participantes segundo as variáveis socioeconômicas. Método: estudo descritivo, de natureza quantitativa, desenvolvido num Multicentro de Saúde de Salvador, Bahia, Brasil, em 2017. Realizou-se análises descritivas, utilizando tabelas contendo frequências absolutas e relativas. Resultados: a amostra foi constituída por 220 participantes com diagnóstico de hipertensão arterial, predomínio de mulheres (78,6\%), com idade maior ou igual a 60 anos $(53,6 \%)$, de cor preta e parda (91,4\%), com companheiro $(77,7 \%)$, renda familiar mensal de um a dois salários mínimos (48,6\%) e escolaridade até o ensino médio completo (55,5\%). Destacaram-se, na amostra, obesidade e sobrepeso $(40,1 \%)$ e circunferência da cintura (CC) e relação cintura-quadril (RCQ) não recomendados. Conclusão: houve predomínio de sobrepeso e obesidade I e II, circunferência de cintura e razão cintura/quadril não recomendadas.
\end{abstract}

Descritores: Obesidade. Antropometria. Hipertensão.

Objective: to describe the anthropometric patterns of hypertensive adults and characterize the participants according to socioeconomic variables. Method: a descriptive study with a quantitative approach was carried out in a bealth multicenter in the city of Salvador, Babia, Brazil, in 2017. Descriptive analyses were carried out with the use of tables containing absolute and relative frequencies. Results: the sample was made up of 220 participants with a bypertension diagnosis. There was a prevalence of women (78.6\%) aged 60 years or older (53.6\%) with black and brown skin color (91.4\%), living with their partners (77.7\%), with a monthly family income of one to two minimum wages (48.6\%), and with education level up to complete high school (55.5\%). Obesity and overweight (40.1\%), and waist circumference (WC) and waist-hip ratio (WHR) over recommended levels stood out in the sample. Conclusion: there was a prevalence of overweight and obesity I and II and waist circumference and waist-hip ratio over recommended levels.

Descriptors: Obesity. Anthropometry. Hypertension.

Objetivo: describir los patrones antropométricos de adultos hipertensos y caracterizar a los participantes según las variables socioeconómicas. Método: estudio descriptivo, de naturaleza cuantitativa, desarrollado en un Multicentro

\footnotetext{
Enfermeira. Doutora em Enfermagem. Professora Adjunta III da Escola de Enfermagem da Universidade Federal da Bahia. Salvador, Bahia, Brasil.ccgspires@uol.com.br Graduanda em Enfermagem da Escola de Enfermagem da Universidade Federal da Bahia. Salvador, Bahia, Brasil.

Enfermeiro. Enfermeiro trainee no Hospital Cárdio Pulmonar. Salvador, Bahia, Brasil.
} 
de Salud de Salvador, Bahía, Brasil, en 2017. Resultados: muestra constituida por 220 participantes con diagnóstico de hipertensión arterial, predominio de mujeres (78,6\%), con edad mayor o igual a 60 años (53,6\%), de color negro y parda (91,4\%), con un compañero (77,7\%), renta familiar mensual de uno a dos salarios minimos (48,6\%) y escolaridad hasta la enseñanza media completa (55,5\%). Se destacaron, en la muestra, obesidady sobrepeso (40, 1\%) y circunferencia de la cintura (CC) y relación cintura-cadera (RCQ) no recomendados. Conclusión: predominio de sobrepeso y obesidad I y II, circunferencia de cintura y razón cintura cadera no recomendadas.

Descriptores: Obesidad. Antropometría. Hipertensión.

\section{Introdução}

A hipertensão é responsável por, pelo menos, 45\% das mortes por doenças cardíacas e 51\% das mortes por acidente vascular cerebral. A maior parte das mortes ocorre em países em desenvolvimento $^{(1-2)}$. A maior incidência de Doenças Cardiovasculares (DCVs), como dislipidemias, hipertensão arterial, diabetes tipo 2 e distúrbios de coagulação, possui como um dos seus fatores agravantes a associação com o excesso de peso ${ }^{(3-5)}$.

A associação entre DCVs e obesidade acarreta um aumento significativo na mortalidade populacional. As medidas antropométricas - circunferência abdominal (CA), circunferência do quadril (CQ) e razão cintura-quadril (RCQ) - são instrumentos que auxiliam na determinação da associação entre excesso de peso e fatores de risco cardiovasculares (FRCVs) ${ }^{(3-5)}$.

As avaliações antropométricas são obtidas por meio de medidas de tamanho, peso e proporções do corpo humano. Elas proporcionam dados objetivos e utilizam técnicas simples, não invasivas, sem risco para o sujeito e com baixo custo. Também fornecem dados comparáveis, uma vez que foi estabelecido o padrão de normalidade para a população ${ }^{(6)}$.

A medida da CA, considerada o índice antropométrico mais representativo da gordura intra-abdominal e de aferição mais simples e reprodutível, é obtida por meio da medida da região central, entre a crista ilíaca e o rebordo costal inferior. Já para a medida da CQ utiliza-se o maior diâmetro entre os trocânteres maiores ${ }^{(3,7-8)}$.

A combinação de massa corporal e distribuição de gordura é, provavelmente, a melhor opção para preencher a necessidade de avaliação clínica. Para o cálculo da RCQ, preconiza-se a divisão da CA pela CQ. A realização das medidas antropométricas pelos enfermeiros auxilia na correlação entre obesidade, distúrbios metabólicos e DCVs ${ }^{(3,7-8)}$.

O aumento da obesidade vem se tornando um grande problema de saúde pública na maior parte dos países desenvolvidos e em desenvolvimento. O Índice de Massa Corporal (IMC) é um dos melhores indicadores de gordura corporal. No entanto, esse índice não distingue massa gordurosa de massa magra e não reflete a distribuição de gordura corporal. Portanto, o ideal é que o IMC seja utilizado em conjunto com outros métodos de determinação de gordura corporal. O ponto de corte para adultos baseia-se na associação entre IMC e doenças crônicas ou mortalidade $\mathrm{e}^{(3)}$.

Para tal, convenciona-se chamar de sobrepeso o IMC de 25 a 29,9 kg/m² para obesidade, o IMC maior ou igual a $30 \mathrm{~kg} / \mathrm{m}^{2}$. Os pontos de corte de $<16 \mathrm{~kg} / \mathrm{m}^{2}$ (baixo peso grave), 16,0-16,9 (baixo peso moderado), 17,0-18,4 (baixo peso leve) também fazem parte da classificação internacional. A associação da medida da CA com o IMC pode oferecer uma forma combinada de avaliação de risco e ajudar a diminuir as limitações de cada uma das avaliações isoladas ${ }^{(3-8)}$.

Ao fazer a busca nas Bases de dados da Biblioteca Virtual em Saúde, utilizando os descritores em saúde obesidade and antropometria and hipertensão and prevenção and controle, nos idiomas português, inglês e espanhol, encontrou-se 20 artigos. Contudo, observa-se uma enorme lacuna de pesquisas que relatem investigações com adultos. Foram encontrados estudos 
que tiveram como participantes idosos e escolares e nenhum foi realizado na região Nordeste do Brasil.

Acredita-se que este estudo contribuirá na disseminação do conhecimento e reflexão sobre a importância da mensuração de dados antropométricos e níveis pressóricos de forma correta e com acurácia, subsidiados por guidelines nacionais e internacionais, com vistas a minimizar os agravos sociais e reduzir os impactos provocados pelo descontrole da hipertensão arterial em pessoas acometidas pela doença.

Diante desse contexto, este estudo objetivou descrever os padrões antropométricos (índice de massa corpórea, circunferência da cintura, circunferência do quadril e razão cintura quadril) de adultos hipertensos e caracterizar os participantes segundo as variáveis socioeconômicas.

\section{Método}

Trata-se de um estudo descritivo de natureza quantitativa, realizado num Multicentro de Saúde de um bairro considerado um dos mais populosos da cidade de Salvador, Bahia, Brasil, de forte influência afrodescendente no ano de 2017. A população alvo do estudo foi constituída por pessoas de ambos os sexos, com diagnóstico médico de Hipertensão Arterial Sistêmica (HAS) (CID: I-10), que frequentavam o Multicentro de saúde. Foi calculada a amostra aleatória simples sem reposição, com a finalidade de encontrar a proporção de indivíduos maiores de 18 anos, com diagnóstico de HAS que frequentam o serviço. Utilizou-se o software STATA versão 11.

Adotou-se a prevalência de HAS em maiores de 18 anos de 25,7\%, obtida com base nos resultados do Vigitel 2014. Admitiu-se um erro amostral de 5\% $(d=0,05)$, sob o nível de confiança de $95 \%$. No denominador foi utilizado o total de pacientes cadastrados no Sistema de Cadastramento e Acompanhamentos de Hipertensos e Diabéticos (HIPERDIA). De acordo com o cálculo, o tamanho da amostra foi de 220 pessoas com diagnóstico médico de HAS.

Foram adotados como critérios de inclusão pessoas com diagnóstico médico de HAS, com idade igual ou maior que 18 anos, que frequentavam o Multicentro de Saúde no período da coleta de dados (de julho a dezembro de 2017) para consultas e dispensação de medicamentos por meio da receita nominal. Foram critérios de exclusão: indivíduos acometidos por distúrbios cognitivos e/ou distúrbios psiquiátricos.

O instrumento de coleta de dados foi constituído de questões fechadas referentes a dados socioeconômicos: idade em anos, sexo, renda familiar mensal em número de salários mínimos, cor/raça autodeclarada, situação conjugal, escolaridade e registro dos dados antropométricos: peso atual, altura, circunferência da cintura, relação cintura-quadril, índice de massa corpórea (IMC).

Os parâmetros da Organização Mundial da Saúde $^{(9)}$ foram adotados para mensuração dos dados antropométricos e definição do índice de massa corpórea. Para determinação da circunferência da cintura (CC), aplicou-se os critérios estabelecidos por estudo que tratou dos fatores de risco para doenças cardiovasculares ${ }^{(10)} \mathrm{em}$ consonância com a International Diabetes Federation $^{(11)}$. Solicitou-se ao usuário que ficasse de pé, braços ao lado do corpo e os pés juntos, em posição ortostática. O local da medida foi no ponto médio entre a última costela flutuante e a crista ilíaca, que foi marcado com um lápis ou caneta. A seguir, a fita métrica inelástica de 1,50 m foi posicionada no ponto médio, circundando o abdome segundo um plano horizontal (sem usar o umbigo como ponto de referência); a medida foi feita no fim de uma expiração normal.

Para os parâmetros da razão cintura/quadril, adotaram-se recomendações estabelecidas ${ }^{8}$, realizando a mensuração no ponto de maior circunferência da região glútea com a fita circundando a parte mais saliente entre a cintura e a coxa. As medidas foram feitas com a menor quantidade de roupa possível, conforme exposto anteriormente. A relação cintura quadril foi determinada pela divisão da circunferência de cintura $(\mathrm{cm})$ pela circunferência do quadril $(\mathrm{cm})$.

Os dados antropométricos foram mensurados por duas bolsistas de iniciação científica devidamente treinadas para a realização do 
procedimento. Os participantes do estudo foram convidados, de forma aleatória, enquanto aguardavam consultas e/ou dispensação de medicamentos. Nesse momento, foi-lhes explicado o objetivo do estudo. Em caso de aquiescência, os participantes foram encaminhados para um local reservado, com vistas a promover a privacidade no momento da entrevista. Após a leitura e assinatura do Termo de Consentimento Livre e Esclarecido (TCLE), iniciou-se a coleta de dados com o preenchimento dos instrumentos e aferição das medidas. Ao final da entrevista, agradecia-se às pessoas que participavam do estudo e informava-se que os resultados seriam divulgados no serviço para profissionais e usuários ao término da pesquisa.

Os dados foram codificados, armazenados e analisados no programa estatístico Statistical Package of Social Science (SPSS), versão 21.0, da plataforma Windows. Inicialmente foram realizadas análises descritivas e exploratórias, para caracterizar a população do estudo. As variáveis estudadas são apresentadas descritivamente em tabela contendo frequências absolutas ( $n$ ) e relativas (\%), média e desvio padrão para idade.

O desenvolvimento do estudo atendeu às normas nacionais e internacionais de ética em pesquisa envolvendo seres humanos.

\section{Resultados}

A amostra foi constituída por 220 hipertensos. A média de idade foi de 59,4 anos, desvio padrão 11,3. Predominou o sexo feminino (78,6\%), renda mensal 1 a 2 salários mínimos (48,6\%), a raça negra (91,4\%), convivência com companheiro(a) $(77,7 \%)$ e nível educacional até o ensino médio $(55,0 \%)$.

Conforme demonstrado na Tabela 1 , no estudo, predominaram pessoas obesas (77,7\%), dentre as quais $37,7 \%$ apresentavam sobrepeso e 40,0\%, obesidade grau I e II. Em 90,0\% dos participantes, a circunferência da cintura não era recomendada (maior ou igual a 90 para homens e > ou igual a 80 para mulheres) e 93,2\% com risco elevado para razão cintura/quadril (maior ou igual a $0,9 \mathrm{~cm}$ para homens e maior ou igual a $0,8 \mathrm{~cm}$ para as mulheres).

Tabela 1 - Proporção de pessoas com hipertensão arterial, segundo padrões antropométricos. Salvador, Bahia, Brasil - $2017(\mathrm{~N}=220)$

\begin{tabular}{|c|c|c|}
\hline Padrões antropométricos & $\mathbf{n}$ & $\%$ \\
\hline \multicolumn{3}{|l|}{ Índice de Massa Corporal (IMC) } \\
\hline Normal $(18,5-24,9)$ & 49 & 22,3 \\
\hline Sobrepeso $(\geq 25-29,9)$ & 83 & 37,7 \\
\hline Obesidade I $(30-34,9)$ e II $(35-39,9)$ & 88 & 40,0 \\
\hline \multicolumn{3}{|l|}{ Circunferência da cintura (IDF) em cm } \\
\hline Recomendado $(<90-\mathrm{M} /<80-\mathrm{F})$ & 22 & 10,0 \\
\hline Não recomendado ( $\geq 90-\mathrm{M} / \geq 80-\mathrm{F})$ & 198 & 90,0 \\
\hline \multicolumn{3}{|l|}{ Razão cintura/quadril (cm/cm) } \\
\hline Recomendado $(<0,9-\mathrm{M} /<0,8-\mathrm{F})$ & 15 & 6,8 \\
\hline Não recomendado ( $\geq 0,9-\mathrm{M} / \geq 0,8-\mathrm{F})$ & 205 & 93,2 \\
\hline
\end{tabular}

Fonte: Elaboração própria.

Nota: M - Sexo masculino; F - Sexo feminino.

\section{Discussão}

Neste estudo verificou-se que a predominância da amostra era do sexo feminino e de faixa etária superior a 60 anos. Esse panorama pode ser justificado devido ao fato de mulheres e idosos buscarem mais frequentemente serviços preventivos de saúde para controle e tratamento 
da hipertensão e expressarem maior percepção de sinais e sintomas indicativos da doença. No caso dos idosos, alterações como o enrijecimento de artérias, decorrentes do processo de envelhecimento, contribuem para o desenvolvimento de hipertensão ${ }^{(12-13)}$.

No que tange à raça/cor autorreferida, a mais prevalente foi a negra (cor preta e parda), refletindo uma das características do bairro onde se realizou a pesquisa, de forte influência da cultura negra, no qual se cultivam as raízes africanas e desenvolve-se um trabalho social que busca resgatar a autoestima do povo negro.

Em relação à renda familiar mensal, escolaridade e situação conjugal, houve predomínio de 1 a 2 salários mínimos, até o ensino médio e com companheira(o), respectivamente. Os resultados encontrados assemelharam-se aos de outros estudos $^{(10,12)}$. A renda familiar e a escolaridade são consideradas fatores indicativos da qualidade de vida populacional, pois influenciam no acesso à educação, na adoção de comportamentos saudáveis e adesão a tratamentos de condições crônicas, como a $\mathrm{HAS}^{(12)}$.

Quanto aos padrões antropométricos da amostra, a grande maioria dos participantes encontrava-se acima do peso, com CC e RCQ não recomendados. Os resultados obtidos neste estudo estão em concordância com os de outros, que demonstram a relação entre excesso de peso e hipertensão ${ }^{(6,13)}$.

Como fatores limitantes do presente estudo, elenca-se a coleta dos dados ter sido realizada em um único Multicentro, localizado num bairro com a maior concentração de afrodescendente do município, com características próprias da cultura local e pela evidência científica de risco para hipertensão arterial. Por se tratar de um estudo do tipo transversal, no qual os desfechos são visualizados em um único momento, não estabelecendo relação de causa e efeito, usa-se de cautela quanto à generalização dos resultados.

\section{Conclusão}

Neste estudo foi possível identificar as características da população de adultos hipertensos, tendo sido encontrada maior proporção de mulheres, idosas, obesas, da raça negra que viviam com companheiro, escolaridade até o ensino médio, baixa renda mensal e elevado risco de desenvolver DCV.

Nos parâmetros antropométricos, houve maior proporção do sobrepeso e obesidade I e II, circunferência de cintura e razão cintura/quadril não recomendadas.

Esse resultado demonstra a importância de formulação de políticas públicas voltadas para a melhora da qualidade de vida dessa população.

Assim, sugere-se a ampliação deste estudo para os demais serviços da atenção básica no município, com vistas ao acompanhamento direcionado no tratamento e na prevenção dessas comorbidades (hipertensão e obesidade) na atenção primária à saúde.

\section{Colaborações:}

1. concepção, projeto, análise e interpretação dos dados: Cláudia Geovana da Silva Pires;

2. redação do artigo e revisão crítica relevante do conteúdo intelectual: Cláudia Geovana da Silva Pires, Maiara da Silva Brandão Rodrigues, Alana de Souza Reis Carneiro e Igor Fenando Lopes Assis;

3. aprovação final da versão a ser publicada: Cláudia Geovana da Silva Pires.

\section{Referências}

1. Malachias MVB, Souza WKSB, Plavnik FL, Rodrigues CIS, Brandão AA, Neves MFT, et al. 7모 Diretriz Brasileira de Hipertensão Arterial. Arq Bras Cardiol. 2016 Set; 107(3).

2. World Health Organization. A global brief on hypertension: silent killer, global public health crisis: World Health Day 2013. Genève (CHE); 2013.

3. Associação Brasileira para o Estudo da Obesidade e Síndrome Metabólica. Diretrizes brasileiras de obesidade. São Paulo; 2016.

4. Boden G, Salehi S. Why does obesity increase the risk for cardiovascular disease? Curr Pharm Des. 2013;19(32):5678-83. 
5. Chung W, Park CG, Ryu OH. Association of a New Measure of Obesity with Hypertension and Health - Related Quality of Life. PloS One. 2016;11(5):155-399.

6. Silva IA, Barros DD, Silva VC, Ferreira EAAP. Antropometria naavaliaçãoda obesidadeabdominal e risco de doenças. REBES. 2014;4(1):41-51.

7. Sociedade Brasileira de Cardiologia. I Diretriz Brasileira de Diagnóstico e Tratamento da Síndrome Metabólica. Arq Bras Cardiol. 2005 Apr;84(supl 1).

8. Porto CC. Semiologia médica. 7a ed. Rio de Janeiro (RJ): Guanabara Koogan; 2014.

9. World Health Organization. Obesity: preventing and managing the global epidemic. Report of a World Health Organization Consultation. Genève (CHE); 2000.

10. Pires CGS, Azevedo SQR, Mussi FC. Fatores de risco cardiovascular em estudantes de enfermagem: elaboração de procedimentos de avaliação. Rev baiana enferm. 2014;28(3):294-302.

11. Alberti KG, Zimmet P, Shaw J. IDF epidemiology task force consensus group. The IDF consensus worldwide definition of metabolic syndrome. Lancet. 2005;366(9491):1059-62.

12. Andrade JMO, Rios LR, Teixeira LS, Vieira FS, Mendes DC, Vieira MA, et al. Influência de fatores socioeconômicos na qualidade de vida de idosos hipertensos. Ciênc Saúde Coletiva. 2014;19(8):3497-504.

13. Bezerra VM, Andrade ACS, César CC, Caiaffa WT. Comunidades quilombolas de Vitória da Conquista, Bahia, Brasil: hipertensão arterial e fatores associados. Cad Saúde Pública. 2013 Jan/ Sep;29(9):1889-902.

Recebido: 9 de junho de 2018 Aprovado: 29 de novembro de 2018 Publicado: 27 de dezembro de 2018

A Revista Baiana de Enfermagem utiliza a Licença Creative Commons - Atribuição-NãoComercial 4.0 Internacional. https://creativecommons.org/licenses/by-nc/4.0/ Este artigo é de acesso aberto distribuído sob os termos da Licença Creative Commons (CC BY-NC). Esta licença permite que outros remixem, adaptem e criem a partir do seu trabalho para fins não comerciais. Embora os novos trabalhos tenham de lhe atribuir o devido crédito e não possam ser usados para fins comerciais, os usuários não têm de licenciar esses trabalhos derivados sob os mesmos termos. 